\title{
Erratum: "The aromatic fluctuation index (FLU): A new aromaticity index based on electron delocalization" [J. Chem Phys. 122, 014109 (2005)]
}

Eduard Matito, Miquel Duran, and Miquel Solà ${ }^{\text {a) }}$

Institut de Química Computacional and Departament de Química, Universitat de Girona, 17071 Girona,

Catalonia, Spain

(Received 6 June 2006; accepted 19 June 2006; published online 2 August 2006)

[DOI: $10.1063 / 1.2222352]$

The $\mathrm{FLU}_{\pi}$ values in the last column of Table I were not correct. They should read as follows. The corrected results yield $r=0.944$ for the plot in Fig. 7, although these changes do not modify any conclusion of the paper.

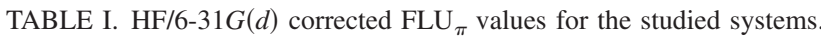

\begin{tabular}{|c|c|c|}
\hline & & $\mathrm{FLU}_{\pi}$ \\
\hline Benzene & M1 & 0.000 \\
\hline Naphthalene & M2 & 0.116 \\
\hline \multirow[t]{2}{*}{ Anthracene } & M3-A & 0.254 \\
\hline & M3-B & 0.024 \\
\hline \multirow[t]{2}{*}{ Naphthacene } & M4-A & 0.355 \\
\hline & M4-B & 0.073 \\
\hline \multirow[t]{2}{*}{ Chrysene } & M5-A & 0.068 \\
\hline & M5-B & 0.185 \\
\hline \multirow[t]{2}{*}{ Triphenylene } & M6-A & 0.026 \\
\hline & M6-B & 0.181 \\
\hline \multirow[t]{2}{*}{ Pyracylene } & M7-A & 0.132 \\
\hline & M7-B & 0.686 \\
\hline \multirow[t]{2}{*}{ Phenanthrene } & M8-A & 0.045 \\
\hline & M8-B & 0.257 \\
\hline \multirow[t]{2}{*}{ Acenaphthylene } & M9-A & 0.117 \\
\hline & M9-B & 0.587 \\
\hline \multirow[t]{2}{*}{ Biphenylene } & M10-A & 0.068 \\
\hline & M10-B & 0.297 \\
\hline \multirow[t]{2}{*}{ Benzocyclobutadiene } & M11-A & 0.196 \\
\hline & M11-B & 1.072 \\
\hline Pyridine & M12 & 0.001 \\
\hline Pyrimidine & M13 & 0.003 \\
\hline Triazine & M14 & 0.000 \\
\hline \multirow[t]{2}{*}{ Quinoline } & M15-A & 0.126 \\
\hline & M15-B & 0.129 \\
\hline Cyclohexane & M16 & $\mathrm{b}$ \\
\hline Cyclohexene & M17 & $\mathrm{b}$ \\
\hline Cyclohexa-1,4-diene & M18 & $\mathrm{b}$ \\
\hline Cyclohexa-1,3-diene & M19 & $\mathrm{b}$ \\
\hline
\end{tabular}

(b) Nonplanar molecules that prevent easy and exact $\sigma-\pi$ separation.

\footnotetext{
${ }^{a)}$ Electronic mail: miquel.sola@udg.es
} 